\title{
S Wave Amplitude Aggregate
}

National Cancer Institute

\section{Source}

National Cancer Institute. S Wave Amplitude Aggregate. NCI Thesaurus. Code C117805.

An aggregate $S$ wave amplitude value based on the measurement of $S$ wave amplitudes

from multiple beats within a single ECG. The method of aggregation, which can vary, is typically a measure of central tendency such as the mean. (CDISC) 